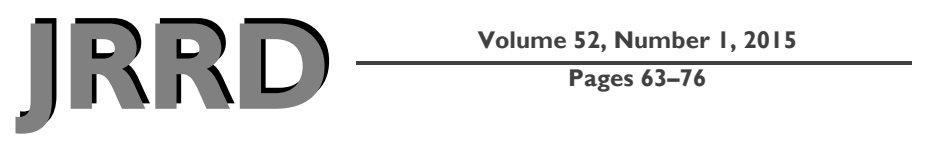

\title{
Design and evaluation of voluntary opening and voluntary closing prosthetic terminal device
}

\author{
Jon W. Sensinger, PhD, PEng; ${ }^{\text {* }}$ James Lipsey, MS, PE; ${ }^{2}$ Ashley Thomas; ${ }^{2}$ Kristi Turner, OTR ${ }^{2}$ \\ ${ }^{1}$ Institute of Biomedical Engineering, University of New Brunswick, Fredericton, Canada, ${ }^{2}$ Center for Bionic Medi- \\ cine, Rehabilitation Institute of Chicago, Chicago, IL
}

\begin{abstract}
Body-powered prostheses use a cable-operated system to generate forces and move prosthetic joints. However, this control system can only generate forces in one direction, so current body-powered prehensor designs allow the user either to voluntarily open or voluntarily close the tongs. Both voluntary opening (VO) and voluntary closing (VC) modes of operation have advantages for certain tasks, and many endusers desire a terminal device (TD) that can switch between the two modes. However, such a TD must maintain the same thumb position (i.e., point of Bowden cable attachment) and movement direction in both modes in order to avoid the need to readjust the harness after every mode switch. In this study, we demonstrate a simple design that fulfills these requirements while allowing the user to switch easily between modes. We describe the design concept, describe a rugged split-hook prototype, provide specifications (size, weight, efficiency, etc.), and present a pilot study in which five subjects with intact arms and two subjects with amputation used the VO and VC splithook prehensor to perform the Southampton Hand Assessment Procedure. Subjects performed an average of 4 to $7(+/-0.2)$ points better when they could choose to switch between modes on a task-by-task basis than when they were constrained to using only $\mathrm{VO}$ or VC modes.
\end{abstract}

Key words: amputation, artificial limb, body-powered prosthesis, grasp, outcomes assessment, prehensor, prosthesis design, terminal device, voluntary closing, voluntary opening.

\section{INTRODUCTION}

Cable-operated mechanisms provide a lightweight yet highly efficient coupling between a user and his or her environment [1]. They work very well for applications such as bicycle brakes, in which a spring holds brake pads apart and the user applies a force through a Bowden cable to close them. The user intuitively knows both the position of the brake pads and feels the force applied via the cable. Such a mechanism could be termed a voluntary closing (VC) mechanism, because the user applies force to voluntarily close the mechanism. In voluntary opening (VO), the closed default state is maintained by a spring and the user applies force to open the mechanism; clothespins are a commonplace example. Even the human hand can be thought of as a cable-operated system, in which pairs of tendons drive each joint. The majority of individuals who have lost their upper limb through amputation, including 68 percent of Vietnam veterans and 38 percent of Operation Iraqi Freedom and Operation Enduring Freedom veterans [2], prefer a cable-operated, body-powered terminal device (TD) over a myoelectric prosthesis (Micera et al., Zecca et al., and Whiteside et al. [3-5]). Body-powered TDs provide improved accuracy

\footnotetext{
Abbreviations: $\mathrm{IOF}=$ index of functionality, $\mathrm{ROM}=$ range of motion, SHAP $=$ Southampton Hand Assessment Procedure, $\mathrm{TD}=$ terminal device, $\mathrm{TH}=$ transhumeral, $\mathrm{VC}=$ voluntary closing, $\mathrm{VO}=$ voluntary opening.

*Address all correspondence to Jon W. Sensinger, PhD, PEng; Institute of Biomedical Engineering, University of New Brunswick, 25 Dineen Dr, PO Box 4400, Fredericton, NB E3B 5A3; 506-458-7094; fax: 506-453-7827.

Email: Sensinger@ieee.org http://dx.doi.org/10.1682/JRRD.2014.03.0087
} 
[6] and extended physiological proprioception [7] in conjunction with rugged design [8-9]. However, as with the bicycle brakes and clothespins described previously and unlike the intact human hand, current body-powered prostheses use only a single cable and enable only one degree of freedom. Body-powered prehensors are thus limited to either VO or VC mode.

Although advantages and disadvantages exist for both types of TDs, most body-powered prosthesis users chose VO. The advantage of $\mathrm{VO}$ is that once the object has been grasped, the user does not need to exert force to maintain that grasp; a spring provides a grip (or pinch) force to hold the object. This allows the user to relax and not be concerned about maintaining cable tension while maneuvering his or her TD and the object in space. The pinch force is determined by the spring tension; for the majority of TDs, the spring tension is fixed and is chosen so as to be adequate for most moderately weighted objects used for daily tasks. However, this spring tension may be excessively strong for some tasks and not strong enough for others (e.g., holding heavy, irregularly shaped, or slippery objects). This limits what types of object the user can successfully manipulate. In addition, users must overcome the force of the spring each time they open the TD, which requires unnecessary energy expenditure whenever the user only needs to hold relatively light objects. Some VO prehensors allow the user to adjust spring tension (Frey et al. and Leblanc and Carlson [10-11]) but only over a limited range of forces. In contrast, for VC TDs, the pinch force is generated by the user. A benefit of this design is that the user can apply the appropriate force for every task, from small forces to large forces; thus, the user need only expend the energy required for the given task. However, in most designs, the user must maintain the pinch force and continue to generate this force throughout the entire task, which can cause fatigue. Although some VC TDs have a clutch that enables the user to relax cable tension while holding the object, the clutch must be disengaged at the end of each movement, which requires an additional action. In addition, clutches often wear out. Because both VO and VC TDs are useful for different subsets of tasks, having to choose one or the other type of TD means that body-powered prosthesis users have difficulty being efficient over the range of tasks they are likely to encounter on a daily basis, and many desire TDs that are more broadly functional [12].

Several groups have tried to design a body-powered prehensor that provides both VO and VC modes. Some have designed mechanisms in which the TD transitions between modes over the course of cable excursion [1316]. These TDs are easy to operate because they do not require a switching mechanism, but they can only generate half the pinch force of conventional TDs since the tong must travel twice as far (i.e., in both the open and close directions) for a given range of cable excursion. This fundamental fact of physics seems likely to limit the clinical viability of this class of TD [17].

Two other groups have created TDs that can switch between $\mathrm{VO}$ and $\mathrm{VC}$ modes and can provide the pinch force obtained from conventional TDs. However, the challenge has been to design TDs that are clinically viable. Sullivan and Siong Teh recently produced a prototype based on a gear transmission, but this design is bulky and heavy and has inefficiencies due to the gears [18]. Perhaps more importantly, the Bowden cable attachment site (commonly called the thumb in the prosthetics field) does not remain the same in the two configurations, which requires the end-users to adjust harness tension every time they switch modes in order to capture the limited cable excursion they can generate with their harness. It is more challenging to design a TD in which the end-effector position remains fixed while the Bowden cable attachment changes from an open to a closed position. Veatch has accomplished such a design in a TD termed LESA, but the implementation was again too heavy and bulky to be clinically viable [19]. In addition, in this design, either the lateral or medial tong moves, depending on the mode. In conventional prehensors, the moveable tong is always the lateral (radial) tong because this allows the user a better line-of-site to the moving tong and the object being manipulated. However, despite these acknowledged design limitations, subjects in pilot studies expressed enthusiasm over the central concept of being able to switch between modes [19].

In a recent study that supports this finding, we found that the majority of subjects with an intact arm performing the Southampton Hand Assessment Procedure (SHAP) [20] with both VO and VC TDs expressed a desire to switch between modes on a task-by-task basis [21]. Thus, a strong need exists for a TD that can switch between modes, but clinical viability requires that it must be the same size and weight as conventional TDs, it must maintain the same default Bowden cable attachment position in both modes, the lateral tong must be the moveable tong in both modes, and the mechanism must be simple enough to allow manufacturers to fabricate the TD for a reimbursable cost. 
In this article we present a simple, rugged split-hook design for a TD that can switch between VO and VC modes and that fulfills all of these requirements. A preliminary design for this $\mathrm{VO} / \mathrm{VC} \mathrm{TD}$ has previously been reported at several conferences [22-23]. Here, we describe the basic design concept along with useful supplemental features and compare the specifications of the TD (including weight, size, efficiency, and pinch force) with conventional TDs. Finally, we perform a pilot study in which persons (five with intact arm and two with amputation) performed the SHAP with this TD using four prehensor configurations: VO or VC (assigned in random order), the alternative mode, the mode they preferred for the task (VO or VC), and the non-preferred mode. We found that six out of seven users had substantial improvements in SHAP scores when they were allowed to use their preferred mode on a task-by-task basis. We conclude with a discussion of the limitations of the TD and this study and provide a brief outline of a larger clinical study currently underway.

\section{METHODS}

\section{Terminal Device Design}

\section{Linkage Singularity}

Basic concept. Our goal for a VO/VC mechanism is to provide an output that changes initial position and movement direction while maintaining a constant initial input position and movement direction. A linkage singularity achieves this goal (Figure 1) [24]. A linkage singularity occurs when three joints lie along a line (Figure 1(b)). At the singularity, movement of the input to the left will result in the input moving up or down. However, when the output joint is above the singularity, it is clear that input movement to the left will result in the output moving down (Figure 1(a)), whereas when the output joint is below the singularity, input movement to the left will result in the output moving up (Figure 1(c)). Note that the input direction (movement to the left) and the input position are the same in both cases (indicated by the vertical lines in Figure 1(a) and 1(c)), yet the output link has both a different initial position (above or below the singularity) and movement direction (up or down). This very simple mechanism is thus able to accomplish all of the required design goals for this $\mathrm{VO} / \mathrm{VC} \mathrm{TD}$.
Note that although forces increase as the linkage transitions through the singularity, the TD is not operated in this state - it only operates on either side of the singularity, thus internal forces remain low. A switch is required to guide the linkages through the singularity, allowing the $\mathrm{TD}$ to be quickly switched between $\mathrm{VO}$ and $\mathrm{VC}$ modes.

Figure 2 shows a schematic and picture of the TD, and Figure 3 shows a visualization of how mode switches occur. A more detailed description of the parts shown is also available [24]. A video of the TD in operation is also available. ${ }^{*}$

Optimization. System parameters (including linkage lengths and joint positions) were optimized using SimMechanics (The MathWorks Inc; Natick, Massachusetts) to provide a variety of features. These included (1) keeping the gear ratio close to $1,(2)$ minimizing axial forces in the linkages for a cable force of $280 \mathrm{~N}$ [8], (3) having both the tong and thumb operate over a range of motion (ROM) of $60^{\circ}$ in both modes, (4) having a switch travel that was reasonably sized and close to horizontal to allow easy engagement, and (5) maintaining sufficient distance between linkage pivot and thumb pilot (Figure 2) to allow space for joint bushings. The positions of both linkage pivots, tong pivot, and thumb pilot (Figure 2) were allowed to vary within geometric boundaries to achieve these goals.

Figure 4 shows the gear ratio of the TD in both modes compared with conventional TDs in either VO or VC mode.

\section{Switch}

The VO/VC mechanism should be easily switched into either mode but should not inadvertently transition between modes when forces are applied during use of the TD; i.e., the mechanism must be nonbackdriveable. In order to achieve a nonbackdriveable switch, we used a design similar to that of a push-button quick-release pin. When the switch toggle is pressed, detents in a piston align with the balls, allowing the balls to drop into the detent (Figure 2). This allows the middle cylinder to move with respect to the outer cylinder. The linkage is connected to the middle cylinder through a slot in the outer cylinder (Figure 2). The switch toggle is internally threaded, such that larger extensions can be screwed on

\footnotetext{
*Sensinger J. RIC VO/VC Prosthetic Terminal Device (patent pending). 2014 Jun 25. Available from: http://youtu.be/ OiSsaEtJ3pI?list=UU90Jc8tmyqwZtLrCZvZpFSw
} 
(a)

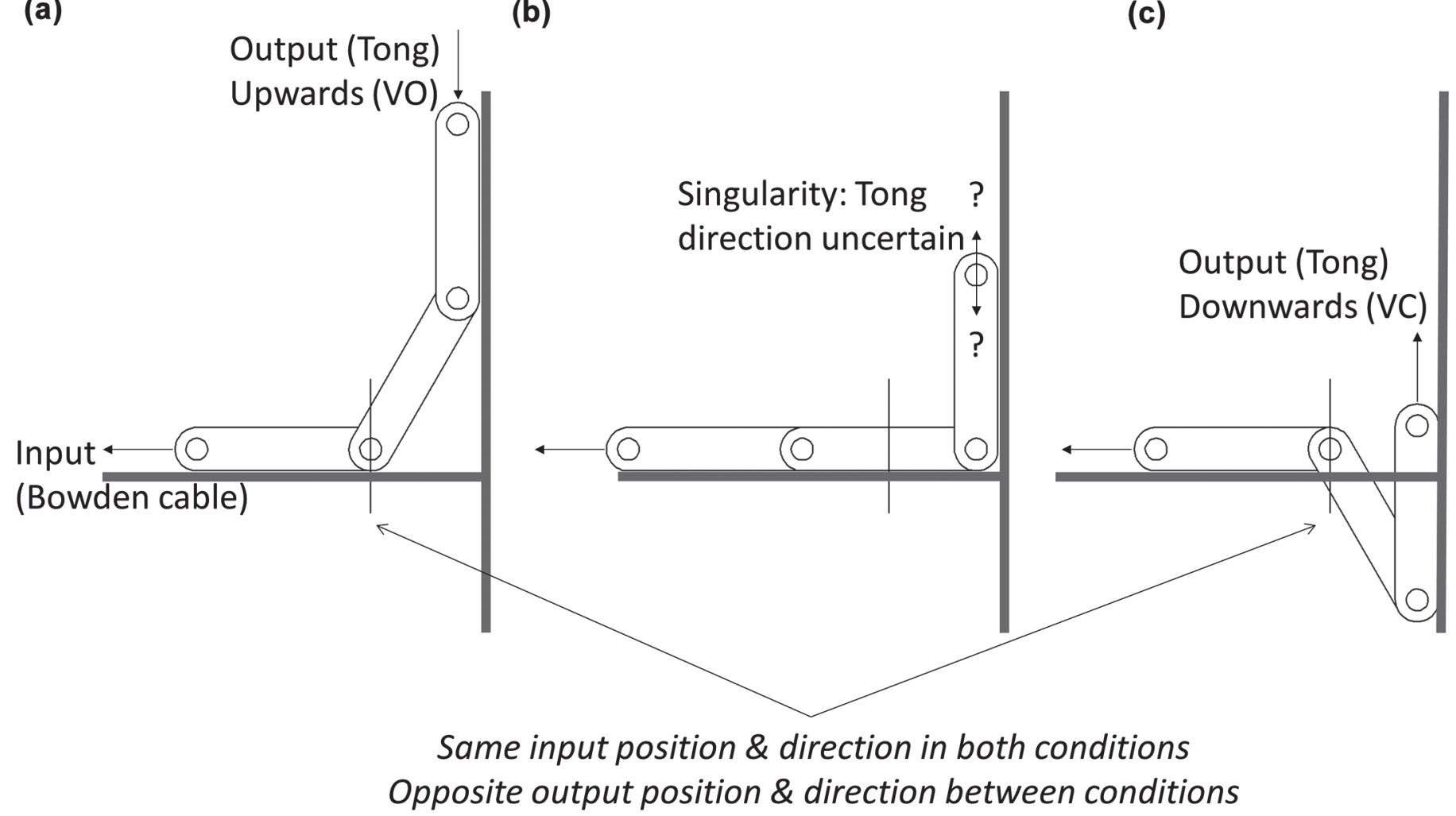

Figure 1.

Linkage singularity achieves bimodal output (initial position and direction) for given input (initial position and direction). (a) Voluntary opening (VO) mode. (b) Passing through singularity. (c) Voluntary closing (VC) mode.

to allow persons with a bilateral amputation to push the switch toggle against their body or another surface to activate the switch.

\section{Spring}

Both $\mathrm{VO}$ and $\mathrm{VC}$ modes require a spring, but the function of this spring in each mode is different. In VO mode, the spring supplies the pinch force, so a moderate spring force is desired. However, in VC mode, the user supplies the pinch force and must overcome the spring force to close the TD, so the minimal spring force that reliably returns the thumb to the default open position is desired. Thus, the desired spring force changes as the mode changes. This may be achieved by attaching one spring to the thumb and another spring to the moving tong. In VO mode, these two linkages rotate in the same direction, and thus the spring forces add together (Figure 5(a)), providing a large net force. In VC mode, the two linkages rotate in opposite directions, subtracting one force from the other (Figure 5(b)) and providing a net force close to 0 .

A bungee cord was used as the tong-attached spring. An off-the-shelf conventional torsion spring was used for the thumb spring, but the measured spring force was consistently lower than that specified. Future designs will use a custom-machined helical spring, which provides higher and more accurate forces for a given size.

\section{Terminal Device Testing}

\section{Summary of Specifications}

All materials were chosen to withstand exposure to water, salt, and debris, and the TD was tested to ensure that no functional damage occurred after 10 drops from a height of $2 \mathrm{~m}$ onto a concrete surface. Table 1 provides a summary of TD parameters. As Table 1 shows, the VO/ VC TD is similar in size to existing TDs. It is also similar in weight to the higher-end TDs such as the Sierra 2 Load 
(a)

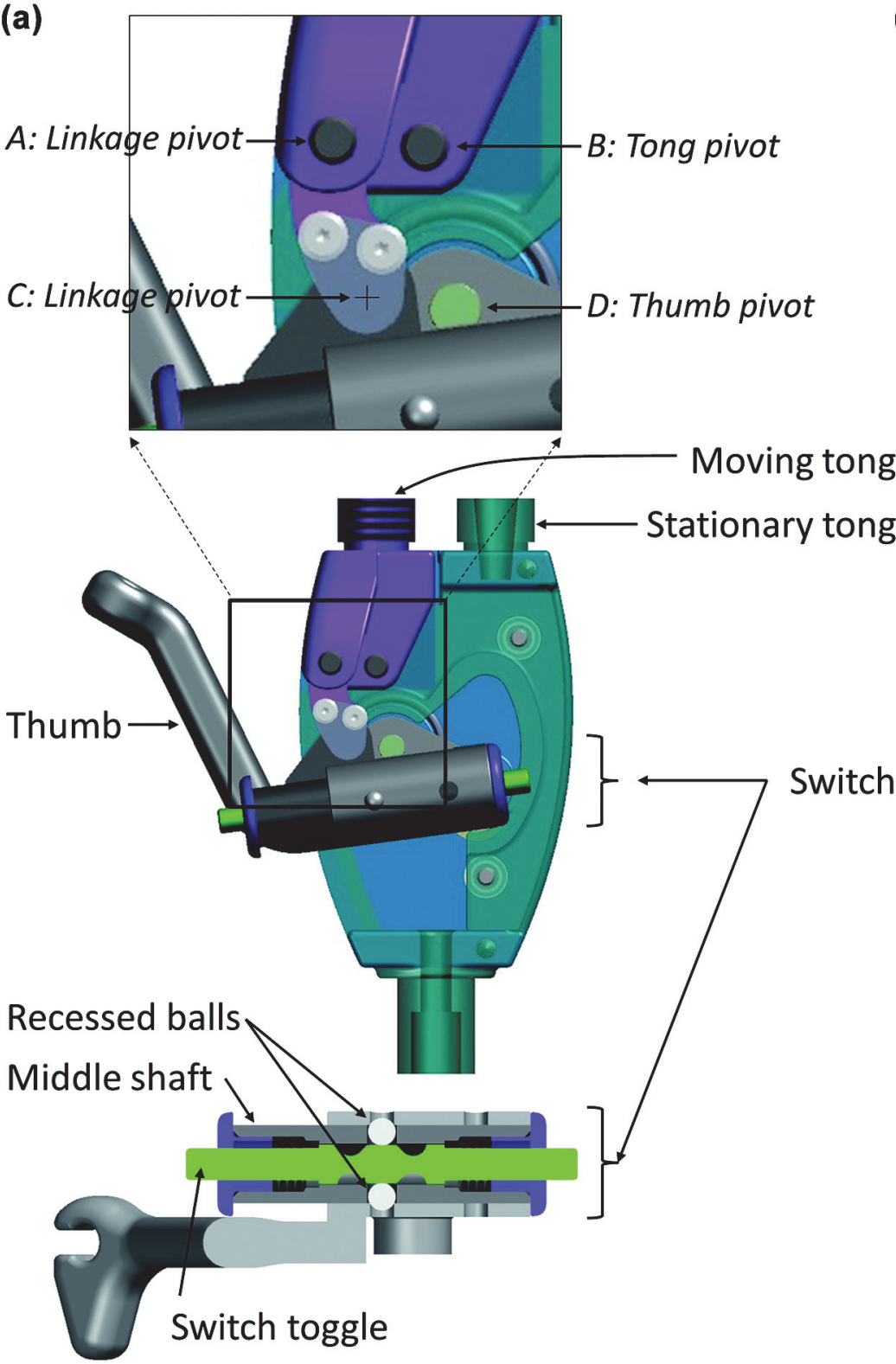

(b)

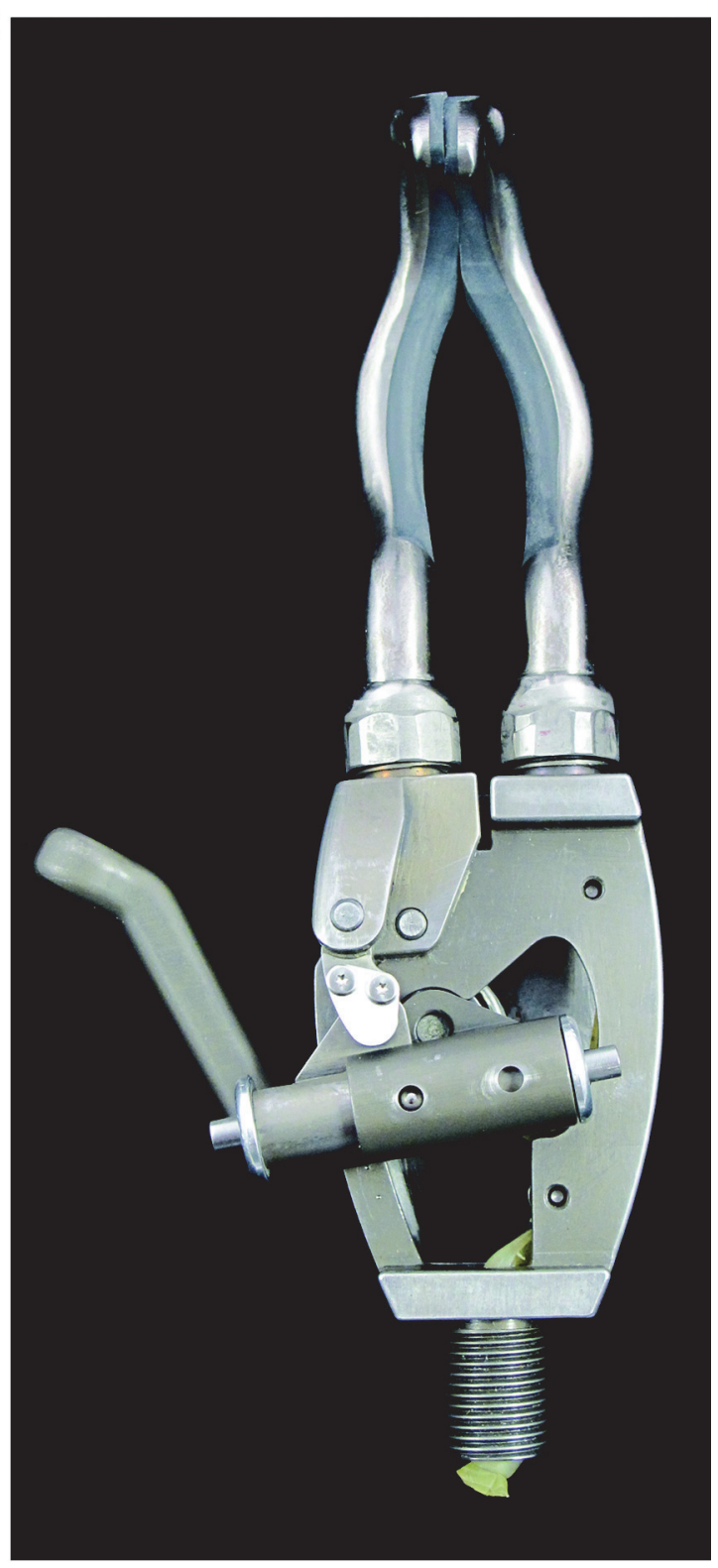

Figure 2.

Voluntary opening (VO) and voluntary closing (VC) design. (a) Dorsal view of design (middle), including linkage (inset, top) and switch (shown in cross-section, bottom). When linkage pivot (C) is to left of thumb pivot (D), terminal device (TD) is in VO mode. When linkage pivot (C) is to right of thumb pivot (D), TD is in VC mode. Position of linkage pivot (C) with respect to thumb pivot (D) is determined by switch position, which is generally locked in place by two recessed balls. Pushing spring-loaded switch toggle in either direction allows balls to drop into recessed groves in switch toggle, allowing middle shaft, which is connected to linkage pivot (C), to move to other position. (b) Embodiment of most recent design (dorsal view).

(Hosmer; Campbell, California). There is a slightly smaller tong opening in VC mode than VO mode because the thumb must have a stop to limit movement in $\mathrm{VC}$ mode, yet the tongs must touch before this stop is reached in VO mode. The TD also performs well ergonomically; cable excursion is identical in both modes and is comparable with other TDs. Switch travel $(12 \mathrm{~mm})$ was increased from original prototype goals $(4 \mathrm{~mm})$ to provide a clearer 

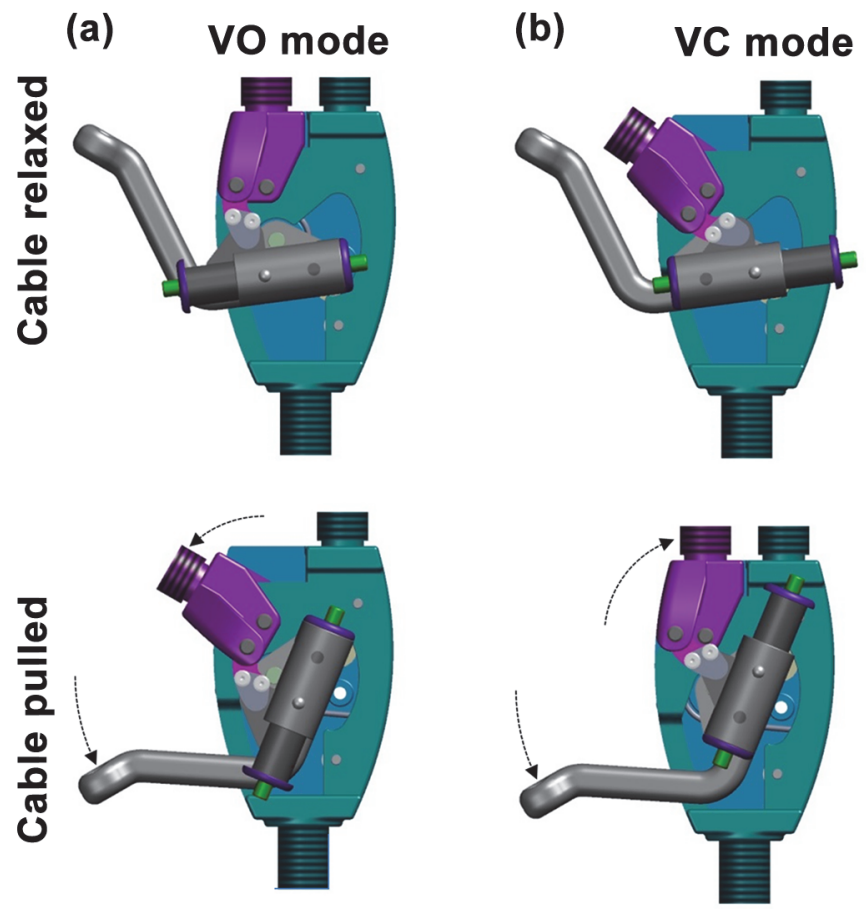

Figure 3.

Terminal device in (a) voluntary opening (VO) and (b) voluntary closing (VC) mode.

indication to the user that a switch had been completed. Switch force is reasonable and higher when transitioning from $\mathrm{VO}$ to $\mathrm{VC}$ mode because the switch must store energy in the tong spring (in order to generate a difference in pinch force between the two modes). Performance metrics are discussed in a separate section.

\section{Performance}

The effort required to operate a TD is an important consideration for both VO and VC TDs. However, the differences between VO and VC TDs make it difficult to compare them. For example, for VO TDs, a large effort is acceptable (since it indicates the TD is exerting large pinch forces), but the TD should convert all of that effort to the pinch force-it should not waste any effort due to TD inefficiency. For VC TDs, however, the TD should require minimum effort to operate over the ROM, since this effort is subtracted from the total user effort to generate the net pinch force. Thus, for VC TDs, a TD that requires less effort to operate is preferable-even if a large portion of that effort is lost to system inefficiencies, although as a general rule it is easier to reduce the effort with more efficient TDs. Force-displacement hysteresis

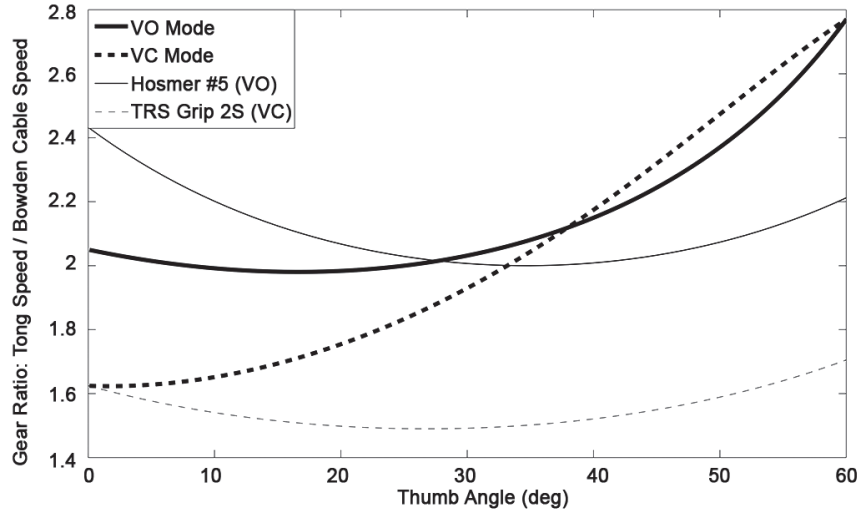

Figure 4.

Gear ratios of voluntary opening (VO) and voluntary closing (VC) prehensor in VO and VC modes and of commercially available $\mathrm{VO}$ and $\mathrm{VC}$ terminal devices (TDs). VO and VC gear ratios are smooth and low, providing user with good control of TD.

is a useful concept that allows comparison of $\mathrm{VO}$ and $\mathrm{VC}$ TDs using the same metric [26]. Figure 6 shows results for the VO/VC TD in VO or VC modes compared with two VO TDs (Model 555 [Hosmer] and Sierra 2 Load) and two VC TDs (APRL [Hosmer] and TRS Grip [TRS; Boulder, Colorado]). The figure displays the ROM of each TD (e.g., the TRS Grip requires the largest Bowden cable displacement and the Sierra 2 Load requires the shortest) and the effort required to operate each TD (e.g., the Model 555 with three rubber bands exerts more pinch force than the weaker mode of the Sierra 2 Load, and the TRS Grip and the APRL require the same amount of effort to operate over their full ROM, although it takes more ROM to close the TRS Grip). In addition, the gap between the two lines, i.e., the hysteresis of the curve, shows the inefficiency of the TD and represents the difference in force when the TD applies and then removes pressure. Note, for example, the larger hysteresis for the Model 555 than for the Sierra 2 Load; this is likely because the Model 555 uses rubber bands, which rub against each other, whereas the Sierra 2 Load uses a coiled torsional spring, which is generally more efficient. Thus, force-displacement hysteresis curves are a useful way to convey a large amount of information about bodypowered prehensors.

From Figure 6, we can see that the VO/VC TD is very efficient, despite the additional linkages in its system. Only $0.08 \mathrm{~J}$ of energy is lost to system inefficiency for each cycle in each mode. This is comparable to the VC 
(a) Cable Tension in VO Mode

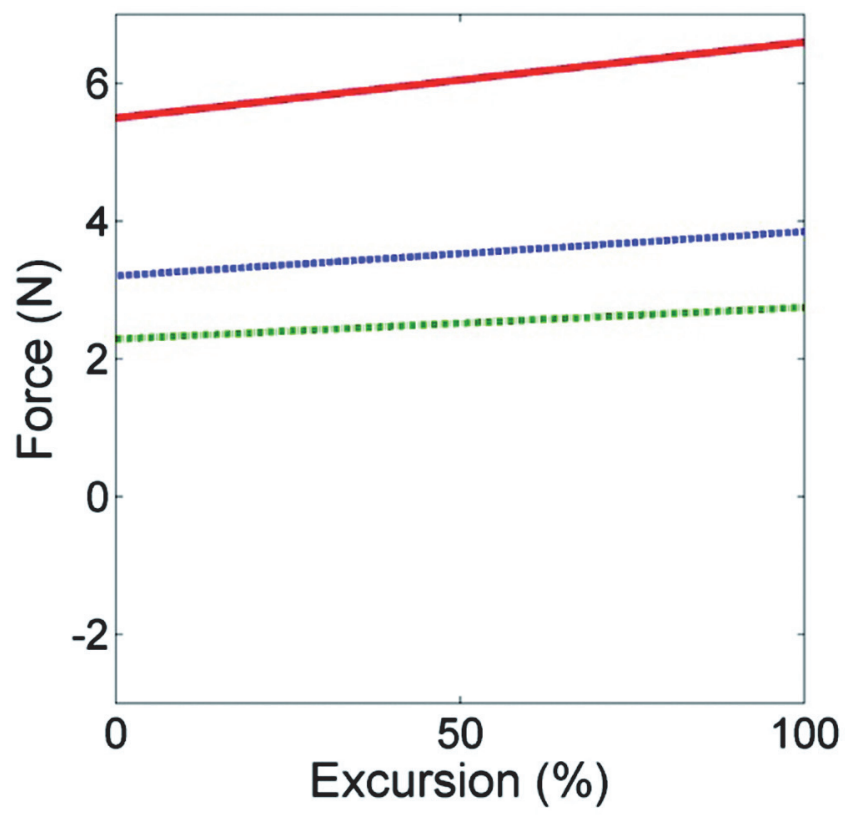

(b) Cable Tension in VC Mode

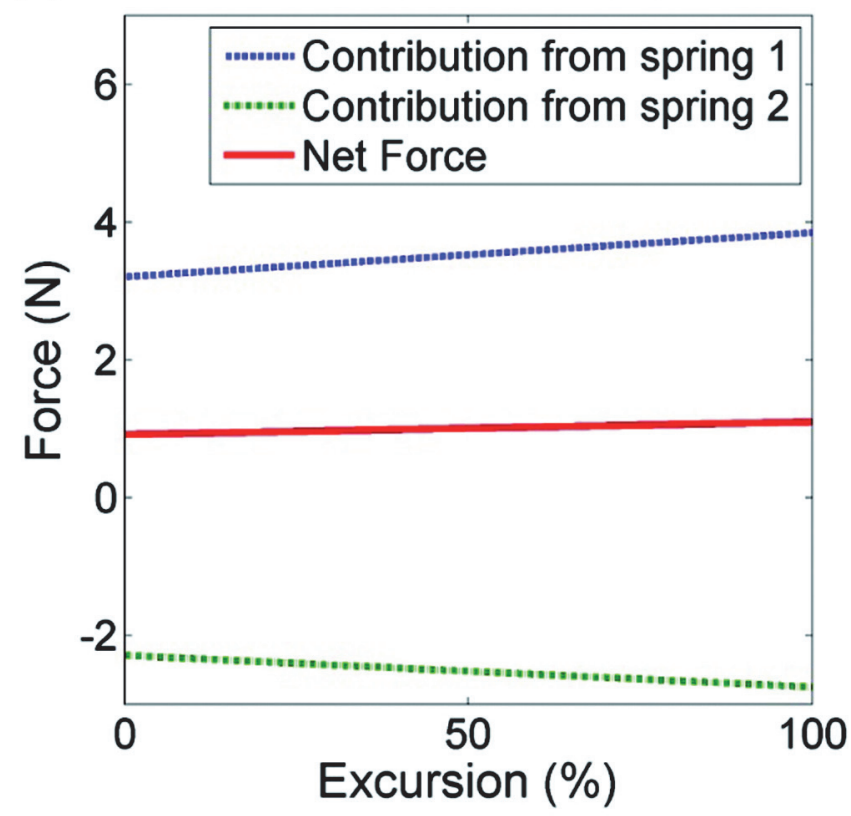

Figure 5.

Forces generated by two springs connected to tong and thumb either (a) add to provide large net pinch force (in voluntary opening [VO] mode) or (b) subtract to provide low net pinch force (in voluntary closing [VC] mode), based on position of switch.

TDs and better than both of the VO TDs (Table 1). We can also see that the ROM is comparable between both modes and in comparison with other TDs. The effort required in $\mathrm{VC}$ mode is only slightly higher than for the TRS Grip and substantially lower than for the ARPL. The effort required in $\mathrm{VO}$ mode is higher than that required in VC mode, demonstrating that the two-spring system is properly adding and subtracting. However, the effort in VO mode (i.e., maximum pinch force) is also substantially lower than the Model 555 or the Sierra 2 Load, even in its weaker setting. Indeed, it is not much higher than the APRL. This key weakness of the current design will be solved by using a stronger helical spring (as mentioned previously) in future versions.

\section{Experiment Materials and Methods}

This experiment used the same methods presented in Berning et al. [21], except where otherwise noted.

\section{Subjects}

Five subjects with an intact arm (age $30 \pm 7 \mathrm{yr}$; 3 females, 2 males; 4 right-hand dominant) and two subjects with amputation (23 yr old female left-handed indi- vidual with a congenital limb absence at the transradial level on the right side and $35 \mathrm{yr}$ old male right-handed individual with a right-side transhumeral [TH]-level amputation) participated in this study. Both subjects with amputation had used a body-powered VO prehensor for at least 6 mo prior to testing and had near normal residuallimb strength. All participants gave informed written consent for this research study, which was approved by the Institutional Review Board at Northwestern University.

\section{Protocol}

Subjects used the VO/VC prehensor to perform the SHAP, which provides a reliable and objective measure of functionality [20,27]. The 26 tasks, selected from activities of daily life, require particular grasps (spherical, power, tip, tripod, lateral, or extension). The tasks are chosen to represent the typical frequency of use of these grasps. Of the tasks, 12 involve moving abstract objects (light and heavy objects, 6 grasp patterns for each). The remaining 14 tasks are activities of daily living (e.g., zipping a zipper or pouring water into a cup). The subject times each task by hitting a timer before starting and after completing the task. Recorded times are entered into the 
Table 1.

Summary of terminal device (TD) parameters.

\begin{tabular}{|c|c|c|c|c|c|c|}
\hline \multirow[b]{2}{*}{ Parameter } & \multirow[b]{2}{*}{ VO/VC TD } & \multirow[b]{2}{*}{ Goal } & \multicolumn{2}{|c|}{ VO Comparisons } & \multicolumn{2}{|c|}{ VC Comparisons } \\
\hline & & & Model 555 & $\begin{array}{c}\text { Sierra } 2 \\
\text { Load }\end{array}$ & APRL & $\begin{array}{l}\text { TRS } \\
\text { Grip }\end{array}$ \\
\hline \multicolumn{7}{|l|}{ Size and Mass } \\
\hline Width $^{*}(\mathrm{~mm})$ & 44 & $<58$ & 42 & 46 & 58 & 40 \\
\hline Thickness (mm) & 38 & $<33$ & 18 & 32 & 33 & 25 \\
\hline Base Length (mm) & 68 & $<70$ & 51 & 53 & 70 & 45 \\
\hline Tong Length from Pivot Point (mm) & 106 & $<100$ & 117 & 101 & 100 & 91 \\
\hline Tong Opening (mm) & $84 / 80$ & $>82^{\dagger}$ & 156 & 70 & 75 & 71 \\
\hline Mass (g) & $352(\mathrm{Al}), 373(\mathrm{Ti})$ & $<136$ & $121(\mathrm{Al}), 241(\mathrm{SS})$ & 354 & 243 & 278 \\
\hline \multicolumn{7}{|l|}{ Ergonomics } \\
\hline Cable Excursion (VO mode) (mm) & 50.8 & 50.0 & 45.2 & 37.4 & NA & NA \\
\hline Cable Excursion (VC mode) (mm) & 50.8 & 50.0 & NA & NA & 50.8 & 62.8 \\
\hline Switch Travel (mm) & $16 / 12^{*}$ & $<4$ & NA & NA & NA & NA \\
\hline Switch Force (N): VO-VC/VC-VO & $46 / 6^{\S}$ & $5^{\mathbb{1}}<\mathrm{F}<20^{* *}$ & NA & NA & NA & NA \\
\hline TD Compliant Slack ${ }^{\dagger \dagger}(\mathrm{mm})$ & 1.8 & 0 & 0 & 0 & 0 & 0 \\
\hline \multicolumn{7}{|l|}{ Performance } \\
\hline Minimum Pinch Force in VO Mode (N) & 6 & 6 & 20 & $16 / 31$ & NA & NA \\
\hline Maximum Cable Tension to Position VC Tong (N) & 12 & $<10$ & NA & NA & 13 & 12 \\
\hline Withstands Maximum Cable Force $(\mathrm{N})$ & - & $840(3 \times 280 \%)$ & - & - & - & - \\
\hline Effort Required to Operate Over ROM (J) & $0.50 / 0.33$ & - & 2.01 & 1.00 & 0.46 & 0.38 \\
\hline Subset of Effort Lost to Inefficiency (J) & $0.08 / 0.08$ & - & 0.25 & 0.13 & 0.08 & 0.08 \\
\hline 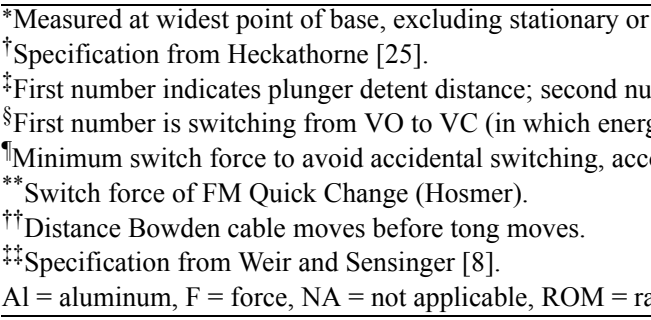 & $\begin{array}{l}\text { moving tong and thumb } \\
\text { nber indicates only dist } \\
\text { y is inserted through sy } \\
\text { rding to International }\end{array}$ & $\begin{array}{l}\text { b. } \\
\text { tance that slider tra } \\
\text { witch into tong spri } \\
\text { Organization for St }\end{array}$ & $\begin{array}{l}\text { avels. } \\
\text { ing); second number is } \\
\text { tandardization } 22523: 2\end{array}$ & $\begin{array}{l}\text { switching fro } \\
06 .\end{array}$ & VC to VO. & \\
\hline
\end{tabular}

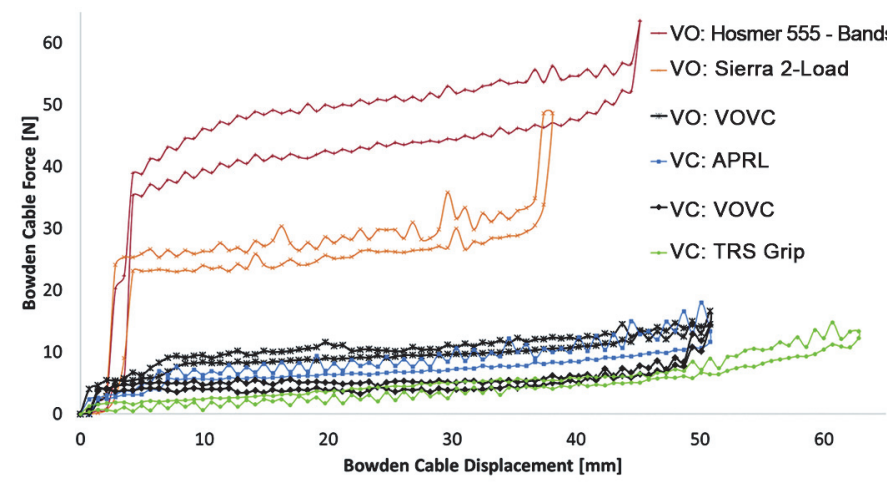

Figure 6.

Force-displacement hysteresis curves for number of terminal devices (TDs), conveying comparative amount of effort needed to operate each TD and relative TD inefficiencies. $V C=$ voluntary closing, $\mathrm{VO}=$ voluntary opening.
SHAP Web site (www.shap.ecs.soton.ac.uk/account.php), which normalizes the data, per task, based on performance and calculates an index of functionality (IOF). An IOF of 100 corresponds with normal function.

Standard practices were followed with the SHAP, including, as the SHAP recommends, allowing the subject to practice each task before performing the timed test. Practice reduced bias toward prehensor order by allowing the subject to develop comfort with the task using each prehensor before completing the timed test.

In contrast with our previous study [21], subjects completed each self-timed task four times before moving on to the next task. For the first repetition, subjects used $\mathrm{VO}$ or VC modes (assigned in random order). In the second repetition, they used the other mode. In the third repetition, subjects used the mode they preferred for this task (VO or VC) and their choice was noted. In the fourth repetition, subjects used their non-preferred mode, in 


\section{Survey Question}

1. What did you like most about the mode in which you open the hook (Voluntary opening)?

2. What did you like most about the mode in which you close the hook (Voluntary closing)?

3. What did you like least about the mode in which you open the hook?

4. What did you like least about the mode in which you close the hook?

5. If you could choose one mode, which would you choose:

$\square$ You open the hook (voluntary open)

Were there any tasks for which you preferred the mode that let you close the hook?

$\square$ No

$O R$

$\square$ Yes. Please describe tasks \& reasons (List of tasks provided on the last page)

$\square$ You close the hook (voluntary close)

Were there any tasks for which you preferred the mode that let you close the hook?

$\square$ No
$\square$ Yes. Please describe tasks \& reasons (List of tasks provided on the last page)

6. What sort of force feedback did you receive from the prosthesis in each mode? Could you feel how hard you were grasping the objects?

7. Did you feel like you had control over the strength of your grasp using the prosthesis in each mode?

8. What are some advantages and disadvantages of this device relative to others you have seen or used?

Figure 7.

Questions listed on subject questionnaire.

order to determine whether any improvement in the third configuration was due to a learning effect.

\section{Survey}

After completing the SHAP, subjects were given a questionnaire to determine their preferences (Figure 7). The questionnaire had eight open-ended questions relating to what subjects liked and disliked about both modes.

\section{Data Processing}

IOF scores were calculated on the SHAP Web site from the recorded task times. The paired difference in IOF score between using the preferred mode and the VO and VC modes, as well as the paired difference in IOF score between using the non-preferred mode and the $\mathrm{VO}$ and $\mathrm{VC}$ modes, was calculated with a one-tail paired Student $t$-test.

\section{RESULTS}

\section{Southampton Hand Assessment Procedure Results}

Both subjects with amputation and four out of the five subjects with an intact arm obtained higher SHAP scores when they could choose their preferred mode on a task-specific basis than when they were forced to use either VO or VC mode (Table 2). Subjects did not have a substantial improvement in the fourth trial, in which they used their nonpreferred mode, which is consistent with previous studies that indicate that learning does not occur across trials [21]. Average ( \pm standard deviation) IOF scores for VO $(50.2 \pm 7.3)$ and VC $(53.8 \pm 11.0)$ were similar but slightly lower than IOF scores in a previous study that used commercially available $\mathrm{VO}$ and VC TDs $(53.6 \pm 10.9$ for $\mathrm{VO}$ and $55.4 \pm 11.5$ for $\mathrm{VC})$ [21], although not enough subjects took part in this pilot study to determine whether that difference is significant.

Subjects typically had a clear preference for most tasks, but for a few tasks (e.g., abstract tripod/tip prehension and tray), subjects felt that both modes performed equally well.

Figure 8 shows a histogram of the level of agreement across subjects per task, where a score of 0 means that all subjects chose VO mode and a score of 1 means that all subjects chose VC mode. As can be seen, subjects had individual mode preferences for the majority of tasks, and there was an overall moderate preference for $\mathrm{VC}$ mode. There was universal agreement in favor of $\mathrm{VC}$ mode for two tasks (jar lid and zipper). A full list of user choices is provided in the Appendix (available online only). 
Table 2.

Index of functionality (IOF) scores for five subjects with intact arm (ND), subject with transradial (TR) amputation, and subject with transhumeral (TH) amputation.

\begin{tabular}{lcrrrrrrrr}
\hline \multicolumn{1}{c}{ IOF Scores } & ND1 & ND2 & ND3 & ND4 & ND5 & Mean \pm SD & $\boldsymbol{p}$-Value & TR1 & TH1 \\
\hline VO & 39 & 59 & 49 & 52 & 52 & $50.2 \pm 7.3$ & - & 40 & 43 \\
VC & 37 & 65 & 48 & 59 & 60 & $53.8 \pm 11.0$ & - & 44 & 47 \\
Preferred Mode & 49 & 56 & 55 & 64 & 63 & $57.4 \pm 6.2$ & - & 51 & 55 \\
Nonpreferred Mode & 44 & 61 & 45 & 51 & 56 & $51.4 \pm 7.2$ & - & 40 & 47 \\
Preferred Mode - VO Mode & 10 & -3 & 6 & 12 & 11 & $7.2 \pm 5.5$ & 0.03 & 11 & 12 \\
Preferred Mode - VC Mode & 12 & -9 & 7 & 5 & 3 & $3.6 \pm 7.0$ & 0.18 & 7 & 8 \\
Nonpreferred Mode - VO Mode & 5 & 2 & -4 & -1 & 4 & $1.2 \pm 3.3$ & 0.25 & 0 & 4 \\
Nonpreferred Mode - VC Mode & 7 & -4 & -3 & -8 & -4 & $-2.4 \pm 5.0$ & 0.20 & -4 & 0 \\
\hline VC = voluntary closing, VO = voluntary opening. & & & & & & & \\
\hline
\end{tabular}

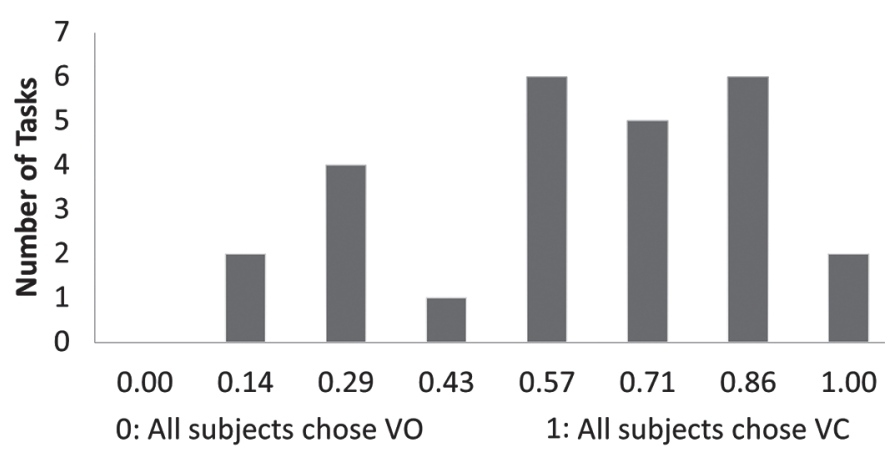

Figure 8.

Histogram of ratios of user preference for voluntary opening (VO) and voluntary closing (VC) modes for 26 Southampton Hand Assessment Procedure tasks. Score of 0 indicates all subjects preferred VO mode, and score of 1 indicates all subjects preferred VC mode. Scores between 0 and 1 indicate that some subjects chose VO mode and some chose VC mode.

\section{Survey Results}

The survey was open-ended, but subjects had similar comments. The number of subjects who provided each answer is provided in parentheses.

Subjects liked VO mode for light, thin, small objects (2); to carry objects over a distance (2); because of the lower mental effort (2); and because it required minimal effort to use (2). They did not think it had enough grip force (4) and did not like the fact that they could not control the grip strength (3). Subjects liked VC mode because they could control the grip force (5), sense the grip force (2), apply large amounts of force (2), and hold oddly shaped objects tightly (2). They did not like the fact that it required more work (4) and felt it was hard to apply constant pressure (4). The majority of subjects (5) would use VC mode if forced to choose, although those subjects would like to use VO mode for a variety of SHAP tasks, including coins, tripod, tip, extension, screwdriver, key, and food cutting. Two subjects would use VO mode if forced to choose, but would prefer to use $\mathrm{VC}$ mode for jar lid, abstract metal cylinder or sphere, button board, key, and zipper.

All subjects reported minimal force control and feedback using VO mode and adequate force control and feedback using VC mode. When asked to compare the TD with others with which they had experience, five subjects reported that it was simple and easy to switch between modes, which seemed an advantageous feature, although one subject clarified that he would need to find more tasks that required $\mathrm{VO}$ mode to make the $\mathrm{VO} / \mathrm{VC}$ TD more useful than a VC TD. Another subject reiterated that it was difficult to maintain cable tension when holding an object in $\mathrm{VC}$ mode while moving the elbow or shoulder (e.g., rotating a screwdriver), which is a known issue for VC TDs [21].

Three subjects noted defects in this particular embodiment of a VO/VC mechanism compared with existing TDs, including that the detachable (Sierra 2 Load) tongs were not perfectly aligned for one subject, that the spring force in VO mode was too weak, and that holding silverware was difficult in both modes. (The original prototype thumb was modeled after the Sierra 2 Load, which has a negligible thumb nub. We have since added a more pronounced nub, similar to that of the Model 5 TD [Hosmer].) 


\section{DISCUSSION}

In this study, we present a design for a body-powered prehensor that is able to switch between $\mathrm{VO}$ and $\mathrm{VC}$ modes using a simple, robust linkage mechanism. This mechanism was embodied in a TD that meets a variety of clinically required design parameters including TD size, weight, function, and ergonomics. The TD performed similarly to existing VO and VC TDs, without substantially increased weight, size, or inefficiency compared with high-end VO and VC TDs such as the Sierra 2 Load or the ARPL.

In a pilot study, both subjects with an intact arm and subjects with amputation performed better on the SHAP when they were able to switch between $\mathrm{VO}$ and $\mathrm{VC}$ modes. Scores on the SHAP using this novel TD in VO and $\mathrm{VC}$ mode were consistent with a recent larger study in which subjects used commercially available VO and VC TDs [21]. Subjects also reported in the user survey that they appreciated the ability to switch between modes.

The performance of the subject with TH amputation is particularly noteworthy. Conventional harnessing for subjects with $\mathrm{TH}$ amputation connects a cable in series with the TD and the elbow. As long as the TD exerts a higher force than the elbow when the elbow is free, and a lower force than the elbow when the elbow is locked, this arrangement is suitable. However, such an arrangement precludes the use of VC TDs, in which the pinch force of the TD is inherently low until it grasps an object. The subject with $\mathrm{TH}$ amputation appreciated the ability to position his TD in VO mode but then operate the TD in VC mode (which he preferred for most tasks).

Although this TD was designed for the size and weight requirements of adults, numerous clinicians have stated that they were interested in providing such a TD to pediatric end-users and requested the TD be scaled down. The linkage concept itself is completely scalable, and future work will involve developing a pediatric version.

\section{STUDY LIMITATIONS}

The largest limitation of the TD was low pinch force in VO mode. Based on the results of a recent study of VO versus VC use [21], we had hypothesized that users would appreciate less force in VO mode if they knew they could generate more force for those few tasks that required it using $\mathrm{VC}$ mode. Said differently, conventional
VO TDs typically generate $13 \mathrm{~N}(3 \mathrm{lb})$ of force. We speculated that most tasks only require $4.5 \mathrm{~N}$ of force, but that in the course of a day, one or two tasks will require $13 \mathrm{~N}$ of force, thus requiring average users to use $13 \mathrm{~N}$ to open the spring for all tasks throughout the day. We accordingly designed the TD to have a light pinch force of $6 \mathrm{~N}$ in VO mode. However, we realized in the course of this study that some tasks, in particular using a knife, are best done in VO mode and require a higher pinch force. In future designs, we will increase this force to $9 \mathrm{~N}$.

This pilot study was limited to five subjects with an intact arm and two subjects with amputation. Since the focus of this study was on the design and performance of the TD, we used questionnaires to supplement these data with clear indications of whether users found the TD useful, as well as important feedback for design improvements. We are currently beginning a field trial of the $\mathrm{VO} /$ VC TD in which persons with amputation will take the TD home for a month, after which they will perform a battery of functional tests using both their conventional $\mathrm{TD}$ and the VO/VC TD.

\section{CONCLUSIONS}

A simple mechanism was developed to enable a user to switch between $\mathrm{VO}$ and $\mathrm{VC}$ modes in a small, rugged body-powered prehensor package. Initial results from end-users indicate the TD has the potential to improve functionality and reduce both the mental and physical effort required for body-powered prosthesis users to interact with their environment on a daily basis.

\section{ACKNOWLEDGMENTS}

\section{Author Contributions:}

Study concept and design: J. W. Sensinger, J. Lipsey, A. Thomas. Acquisition of data: J. W. Sensinger, A. Thomas, K. Turner. Analysis and interpretation of data: J. W. Sensinger.

Drafting of manuscript: J. W. Sensinger.

Critical revision of manuscript for important intellectual content: J. Lipsey, K. Turner.

Statistical analysis: J. W. Sensinger.

Obtained funding: J. W. Sensinger.

Administrative, technical, or material support: J. W. Sensinger. Study supervision: J. W. Sensinger.

Financial Disclosures: The authors have declared that no competing interests exist. 
Funding/Support: This material was based on work supported by the U.S. Department of Education, National Institute for Disability and Rehabilitation Research (grant H133G120059).

Additional Contributions: The authors thank Laura Miller and Todd Kuiken for advice regarding the design of the TD and Ann Barlow for editing the manuscript.

Institutional Review: The authors received human subjects approval from the Northwestern University Institutional Review Board (Chicago, Illinois). All participants gave informed consent.

Participant Follow-Up: The authors do not plan to inform participants of the publication of this study. However, participants have been encouraged to check the study Web site for updated publications.

Disclaimer: This article does not represent the policy of the U.S. Department of Education and does not constitute endorsement by the U.S. Government.

\section{REFERENCES}

1. Schiele A, Letier P, Van der Linde RQ, Van der Helm F. Bowden cable actuator for force-feedback exoskeletons. Proceedings of the 2006 IEEE/RSJ International Conference on Intelligent Robots and Systems; 2006 Oct 9-15; Beijing, China. p. 3599-3604.

2. McFarland LV, Hubbard Winkler SL, Heinemann AW, Jones M, Esquenazi A. Unilateral upper-limb loss: Satisfaction and prosthetic-device use in veterans and servicemembers from Vietnam and OIF/OEF conflicts. J Rehabil Res Dev. 2010;47(4):299-316. [PMID:20803400] http://dx.doi.org/10.1682/JRRD.2009.03.0027

3. Micera S, Carpaneto J, Raspopovic S. Control of hand prostheses using peripheral information. IEEE Rev Biomed Eng. 2010;3:48-68. [PMID:22275201] http://dx.doi.org/10.1109/RBME.2010.2085429

4. Zecca M, Micera S, Carrozza MC, Dario P. Control of multifunctional prosthetic hands by processing the electromyographic signal. Crit Rev Biomed Eng. 2002;30(4-6):459-85. [PMID:12739757]

http://dx.doi.org/10.1615/CritRevBiomedEng.v30.i456.80

5. Whiteside SR, Alaimo J, Barringer WJ, Beiswenger WD, Bulgarelli T, Hentges CJ, Lin RS, Miller TE, Parr RG, Reynolds JH, Stills ML, Hoxie LO, Carter CA, Greenberg $\mathrm{S}$, Sawtell EA. Practice analysis task force. Alexandria (VA): American Board for Certification in Orthotics and Prosthetics; 2000.

6. Vodovnik L, Rebersek S. Information content of myo-control signals for orthotic and prosthetic systems. Arch Phys Med Rehabil. 1974;55(2):52-56. [PMID:4272626]

7. Simpson DC. The choice of control system for the multimovement prosthesis: Extended physiological proprioception (E.P.P). In: Herberts P, Kadefors R, Magnusson R, Peterson I, editors. The control of upper-extremity prosthe- ses and orthoses. Springfield (IL): Charles Thomas; 1974. p. $146-50$.

8. Weir RF, Sensinger JW. Design of artificial arms and hands for prosthetic applications. In: Kutz M, editor. Biomedical engineering and design handbook. 2nd ed. New York (NY): McGraw-Hill; 2009. p. 537-98.

9. Fryer CM, Stark GE, Michael JW. Body-powered components. In: Smith DG, Michael JW, Bowker JH, editors. Atlas of amputations and limb deficiencies: Surgical, prosthetic, and rehabilitation principles. Rosemont (IL): American Academy of Orthopaedic Surgeons; 2004. p. 131-43.

10. Frey DD, Carlson LE, Ramaswamy V. Voluntary-opening prehensors with adjustable grip force. J Prosthet Orthot. 1995;7(4):124-31. http://dx.doi.org/10.1097/00008526-199507040-00004

11. Leblanc MA, Carlson LE. Adjustable prehension device (APD) for prosthetic hooks. Proceedings of the International Conference on Prosthetics and Orthotics; 1992; Chicago, IL. p. 67.

12. Atkins D, Heard DC, Donovan WH. Epidemiologic overview of individuals with upper-limb loss and their reported research priorities. J Prosthet Orthot. 1996;8(1):2-11. http://dx.doi.org/10.1097/00008526-199600810-00003

13. Meeks D, Leblanc M. Preliminary assessment of three new designs of prosthetic prehensors for upper limb amputees. Prosthet Orthot Int. 1988;12(1):41-45. [PMID:3399368]

14. Leblanc MA, Parker D, Nelson C. New designs for prosthetic prehensors. Proceedings of the 9th International Symposium on External Control of Human Extremities; 1987 Aug; Dubrovnik, Yugoslavia. p. 475-81.

15. Procter S, Leblanc M. Clinical evaluation of a new design prosthetic prehensor. J Prosthet Orthot. 1991;3(2):79-83. http://dx.doi.org/10.1097/00008526-199100320-00003

16. Kuniholm J. Body-powered hook. The Open Prosthetics Project [Internet]. Available from:

http://openprosthetics.org/body-powered

17. Sensinger JW. Voluntary opening-closing terminal device design. Proceedings of the 13th International Conference on Prosthetics and Orthotics; 2010; Leipzig, Germany.

18. Sullivan T, Siong Teh K. Design and fabrication of a hybrid body-powered prosthetic hand with voluntary opening and voluntary closing capabilities. Proceedings of the ASME 2011 International Mechanical Engineering Congress and Exposition; 2011 Nov 11-17; Denver, CO. p. 155-62.

19. Veatch BD. A combination $\mathrm{VO} / \mathrm{VC}$ terminal device with variable mechanical advantage. New Orleans (LA): American Academy of Orthotics and Prosthetics; 2004.

20. Light CM, Chappell PH, Kyberd PJ. Establishing a standardized clinical assessment tool of pathologic and prosthetic hand function: Normative data, reliability, and validity. Arch Phys Med Rehabil. 2002;83(6):776-83. 


\section{[PMID:12048655]}

http://dx.doi.org/10.1053/apmr.2002.32737

21. Berning K, Cohick S, Johnson R, Miller LA, Sensinger JW. Comparison of body-powered voluntary opening and voluntary closing prehensor for activities of daily life. J Rehabil Res Dev. 2014;51(2):253-61. [PMID:24933723] http://dx.doi.org/10.1682/JRRD.2013.05.0123

22. Sensinger JW, Cohick S, Sutton L, Thomas A, Lipsey J. Voluntary-opening/voluntary-closing body-powered terminal device provides cosmesis and function. Proceedings of the 14th World Congress of the International Society for Prosthetics and Orthotics; 2013 Feb 4-7; Hyderabad, India.

23. Thomas A, Lipsey J, Sensinger J. Voluntary-opening/voluntary-closing body-powered terminal device provides new level of functionality. Proceedings of the 40th Academy Annual Meeting and Scientific Symposium of the American Academy of Orthotists and Prosthetists; 2014 Feb 26-Mar 1; Chicago, IL.

24. Sensinger JW, inventor; Rehabilitation Institute of Chicago, assignee. Gripping device with switchable opening modes. United States patent US20140081425 A1. 2014 Mar 20.

25. Heckathorne CW. Components for electric-powered systems. In: Smith DG, Michael JW, Bowker JH, editors. Atlas of amputations and limb deficiencies: Surgical, prosthetic, and rehabilitation principles. Rosemont (IL): American Academy of Orthopaedic Surgeons; 2004. p. 145-72.

26. Smit G, Plettenburg DH. Efficiency of voluntary closing hand and hook prostheses. Prosthet Orthot Int. 2010;34(4):
411-27. [PMID:20849359]

http://dx.doi.org/10.3109/03093646.2010.486390

27. Miller LA, Swanson S. Summary and recommendations of the Academy's State of the Science conference on upper limb prosthetic outcome measures. J Prosthet Orthot. 2009;21(4S):83-89.

http://dx.doi.org/10.1097/JPO.0b013e3181ae974d

Submitted for publication March 27, 2014. Accepted in revised form November 10, 2014.

This article and any supplementary material should be cited as follows:

Sensinger JW, Lipsey J, Thomas A, Turner K. Design and evaluation of voluntary opening and voluntary closing prosthetic terminal device. J Rehabil Res Dev. 2015;52(1):63-76.

http://dx.doi.org/10.1682/JRRD.2014.03.0087

ResearcherID: Jon W. Sensinger, PhD, PEng: C-50122014; Ashley Thomas: C-1277-2015; Kristi Turner, OTR: C-1305-2015

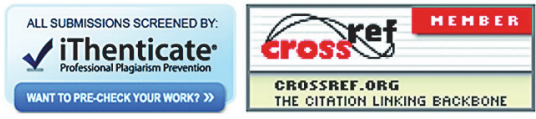


